\title{
NB1 mediates surface expression of the ANCA antigen proteinase 3 on human neutrophils
}

\author{
Sibylle von Vietinghoff, ${ }^{1}$ Gisela Tunnemann, ${ }^{2}$ Claudia Eulenberg, ${ }^{1}$ Maren Wellner, ${ }^{1}$ M. Cristina Cardoso, ${ }^{2}$ Friedrich C. Luft, ${ }^{1,2}$ \\ and Ralph Kettritz ${ }^{1}$ \\ ${ }^{1}$ Franz Volhard Clinic, Department of Nephrology and Hypertension, Medical Faculty of the Charité, HELIOS-Klinikum, Berlin, Germany; ${ }^{2}$ Max-Delbrück Center \\ for Molecular Medicine, Berlin, Germany
}

Antineutrophil cytoplasmic antibodies (ANCAs) with specificity for proteinase 3 (PR3) are central to a form of ANCAassociated vasculitis. Membrane PR3 (mPR3) is expressed only on a subset of neutrophils. The aim of this study was to determine the mechanism of PR3 surface expression on human neutrophils. Neutrophils were isolated from patients and healthy controls, and hematopoietic stem cells from cord blood served as a model of neutrophil differentiation. Surface expression was analyzed by flow cytometry and confocal microscopy, and proteins were analyzed by Western blot experiments. Neutrophil subsets were separated by magnetic cell sorting. Transfection experiments were carried out in HEK293 and HL60 cell lines. Using neutrophils from healthy donors, patients with vasculitis, and neutrophilic differentiated stem cells we found that mPR3 display was restricted to cells expressing neutrophil glycoprotein NB1, a glycosylphosphatidylinositol (GPI)-linked surface receptor. mPR3 expression was decreased by enzymatic removal of GPI anchors from cell membranes and was absent in a patient with paroxysmal nocturnal hemoglobinuria. PR3 and NB1 coimmunoprecipitated from and colocalized on the neutrophil plasma membrane. Transfection with NB1 resulted in specific PR3 surface binding in different cell types. We conclude that PR3 membrane expression on neutrophils is mediated by the NB1 receptor. (Blood. 2007;109:4487-4493)

ㄷ) 2007 by The American Society of Hematology

\section{Introduction}

Antineutrophil cytoplasmic autoantibodies (ANCAs) are central in the ANCA-associated vasculitis syndromes. The neutrophil serine protease proteinase 3 (PR3) is a major autoantigen for ANCA. ${ }^{1}$ Binding of PR3-ANCA to cytokine-primed neutrophils results in respiratory burst activity, increased adhesion, degranulation, and dysregulated apoptosis of neutrophils in vitro. ${ }^{2}$ No suitable animal model for PR3-ANCAassociated vasculitis is available. However, Pfister et $\mathrm{al}^{3}$ recently demonstrated increased dermal inflammation in mice after passive PR3-ANCA transfer. PR3 has also been termed myeloblastin because of its early expression and importance in neutrophil development. ${ }^{4}$ PR3 is stored in primary and secondary granules and is also found in secretory vesicles. ${ }^{5}$ Although all neutrophils contain intracellular PR3, only a subset displays the protein on the plasma membrane allowing neutrophilANCA interaction. ${ }^{6}$ Cytokine-priming increases the amount of mPR3 without affecting the percentage of mPR3-positive cells. In the general population, this percentage varies from 0 to $100 \%$. The percentage of mPR3-expressing cells is stable in a given person and strongly influenced by genetic variance. ${ }^{6-8}$ The mean percentage of mPR3positive neutrophils is increased in patients with ANCA-associated vasculitis, and a higher percentage predicts an unfavorable clinical course. ${ }^{9-12}$ In contrast to the importance of mPR3, little is known about the mechanism of its membrane display. Hydrophobic insertion has been proposed in a system of reconstituted lipid bilayers. ${ }^{13} \mathrm{mPR} 3$ associates with lipid raft proteins, such as integrins and the Fc $\gamma$ receptor. ${ }^{14}$ However, how PR3 is physically presented and how the unique feature of a fixed percentage of mPR3-positive neutrophils is mediated is not known.
The human neutrophil antigen NB1, also known as CD177, human neutrophil antigen $2 \mathrm{~A}$, or polycythemia rubra vera-1 (PRV1), is an approximately 50-kDa glycoprotein. The molecule was first discovered in patients with alloimmune neonatal neutropenia and was later implicated in the pathophysiology of PRV. ${ }^{15,16}$ NB1 belongs to the urokinase plasminogen activator receptor/CD59/ Ly-6 snake toxin superfamily. ${ }^{17}$ These receptors are inserted into the plasma membrane via a glycosylphosphatidylinositol (GPI) linker. Similar to PR3, NB1 shows a bimodal expression pattern with expression on $0 \%$ to $100 \%$ of neutrophils in the population. ${ }^{18}$ We encountered a patient with PRV who kindled our interest in this protein. Our findings caused us to test the hypothesis that NB1 serves as the receptor for PR3 neutrophil membrane display.

\section{Patients, materials, and methods}

\section{Patients and probands}

Neutrophils were obtained from healthy volunteers. Patients with ANCAassociated vasculitis were recruited from our inpatient and outpatient departments. They were diagnosed according to the criteria of the Chapel Hill Consensus Conference and the American College of Rheumatology. ${ }^{19,20}$ Umbilical cord blood was obtained from healthy term deliveries at this hospital. The study was carried out according to the principles of the Declaration of Helsinki and written informed consent was obtained from all subjects or caretakers prior to the studies after approval by the Institutional Review Board of the Charite University Medicine Berlin (Zentrale Ethikkommission.)
Submitted October 30, 2006; accepted January 12, 2007. Prepublished online as Blood First Edition Paper, January 23, 2007; DOI 10.1182/blood-2006-10055327.

The online version of this article contains a data supplement.
The publication costs of this article were defrayed in part by page charge payment. Therefore, and solely to indicate this fact, this article is hereby marked "advertisement" in accordance with 18 USC section 1734.

(C) 2007 by The American Society of Hematology 


\section{Antibodies}

PR3 was detected using CLB12.8. MAB (CLB, Amsterdam, Netherlands), 4A5 and 6A6 (Wieslab, Lund, Sweden), and MCPR3-2 (Dianova, Hamburg, Germany). The NB1 antibody was clone MEM166 (BD Pharmingen, San Diego, CA; BioLegend, San Diego, CA). Anti-CD11b, CD18, and CD66b were from Immunotech (Krefeld, Germany) and CD16b was from Cymbus Biotech (Hants, United Kingdom). Anti-MPO was from Acris Antibodies (Hiddenhausen, Germany), and antielastase and secondary FITC and PE-conjugated $\mathrm{F}(\mathrm{ab})$ fragments of goat anti-mouse IgG were from DAKO (Hamburg, Germany). Cy5-conjugated donkey anti-mouse IgG was from Jackson Immunoresearch (Newmarket, United Kingdom), HRP-labeled goat anti-mouse $\operatorname{IgG}$ was from Santa Cruz Biotechnology (Santa Cruz, CA).

\section{Neutrophil isolation and separation of mPR3- and mNB1-positive and-negative cells}

Neutrophils were isolated from heparinized whole blood by red blood cell sedimentation with dextran 1\%, followed by Ficoll-Hypaque density gradient centrifugation and hypotonic erythrocyte lysis as described. ${ }^{7}$ Cell viability was detected in every cell preparation by trypan blue exclusion and exceeded $99 \%$. The neutrophil percentage in the suspension was greater than $95 \%$ by Wright-Giemsa staining. For separation of subpopulations, isolated neutrophils were stained for PR3 (CLB12.8) or NB1 (MEM166), respectively, labeled with rat anti-mouse IgG microbeads (Miltenyi Biotech, Bergisch-Gladbach, Germany), and separated with magnetic cell sorting LD columns (Miltenyi Biotech) according to the manufacturer's instructions as described. ${ }^{21}$ Purity was assessed by flow cytometry and was $90 \% \pm 5 \%$.

\section{Isolation and culture of hematopoietic CD $34^{+}$progenitors from umbilical cord blood}

Procedures were essentially as described. ${ }^{11}$ Mononuclear cells from heparinanticoagulated cord blood were obtained by centrifugation over a LSM1077 (PAA, Pasching, Austria) gradient $(800 \mathrm{~g}, 30$ minutes). Cells were washed and stained with direct $\mathrm{CD} 34^{+}$progenitor isolation kit (Miltenyi Biotech) and sorted according to the manufacturer's instructions. Cells were cultivated in stem span serum-free medium (Stem Cell Technologies, Vancouver, Canada) supplemented with penicillin/streptomycin, $100 \mathrm{ng} / \mathrm{mL}$ $\mathrm{SCF}, 20 \mathrm{ng} / \mathrm{mL}$ TPO, and $50 \mathrm{ng} / \mathrm{mL}$ FLT3-L (PeproTech, London, United Kingdom) for expansion. Neutrophil differentiation was in RPMI with $10 \%$ FCS and $10 \mathrm{ng} / \mathrm{mL}$ G-CSF (PeproTech). Light microscopy was performed using a Zeiss Axioplan II microscope equipped with a Plan Apochromat $100 \times / 1.4$ oil-immersion objective lens, and image acquisition was performed with a Sensicam 120 using Axiovision 3.0. software (all from Carl Zeiss, Jena, Germany).

\section{Flow cytometry}

Flow cytometry was used as described previously to evaluate the membrane protein expression. ${ }^{21}$ If indicated, cells were stimulated with $2 \mathrm{ng} / \mathrm{mL}$ TNF- $\alpha$ (Genzyme, Rüsselsheim, Germany) or $10^{-9} 10^{-8}$, and $10^{-6} \mathrm{M}$ $N$-formyl-Met-Leu-Phe (fMLP; Sigma, Deisenhofen, Germany) for 20 minutes at $37^{\circ} \mathrm{C}$. Samples were incubated on ice for antibody binding, washed, and counted using a FACScan (Becton Dickinson, Heidelberg, Germany). Ten thousand events per sample were collected and analyzed with Cell-quest Pro software (Becton Dickinson).

\section{Cell lysis, SDS-PAGE, Western Blot, and immunoprecipitation}

Cells were incubated on ice in lysis buffer $(20 \mathrm{mM}$ Tris $\mathrm{HCl}$ pH 8.8, 138 $\mathrm{mM} \mathrm{NaCl}, 10 \%$ Glycerol, 2 mM EDTA, 1\% Triton X-100, 1\% NP40 supplemented with proteinase inhibitors $[10 \mu \mathrm{g} / \mathrm{mL}$ Quercetin, $10 \mu \mathrm{g} / \mathrm{mL}$ Leupeptin, $0.1 \mathrm{mM}$ Aprotinin, $5 \mathrm{mM}$ iodoacetamid), $0.2 \mathrm{mM} \mathrm{Na} \mathrm{VO}_{4}, 20$ $\mathrm{mM} \mathrm{NaF}$, and $1 \mathrm{mM} \mathrm{PMSF}$ ) for 10 minutes, insoluble material was pelleted, and samples were boiled without $\beta$-mercaptoethanol and run in $10 \%$ SDS-polyacrylamide gel electrophoresis (PAGE) gels. Protein was transferred to PVDF-WB membranes (Roche, Mannheim, Germany), and detected by enhanced chemiluminescence-WB reagent (Pierce, Rockford, IL) on hyperfilm (GE Healthcare, Chalfont St Giles, United Kingdom). For immunoprecipitation, the supernatant of $10^{7}$ phospholipase C-treated neutrophils was precleared with protein-A-sepharose beads (GE Healthcare), rotated overnight with $5 \mu \mathrm{g}$ antibody at $4^{\circ} \mathrm{C}$, followed by the precipitation with sepharose for 2 hours. Samples were spun down, washed extensively, boiled, and subjected to SDS-PAGE.

\section{Cleavage of glycosylphosphatidylinositol (GPI) linkers by phospholipase $\mathrm{C}$}

Phospholipase C from Bacillus cereus was obtained from Sigma. Cells were incubated with $0.1 \mathrm{U} / \mathrm{mL}$ at $37^{\circ} \mathrm{C}$ for 2 hours.

\section{Confocal microscopy}

Live cells were analyzed immediately after staining with FITC- or Cy5-labeled antibodies using a Zeiss LSM 510 Meta mounted on an Axiovert $200 \mathrm{M}$ using a $63 \times$ phase-contrast plan-apochromat oil objective (numerical aperture [NA] 1.4) at room temperature. Acquisition settings for all images were UV/488/543/633, and specific parameters for the fluorophores were FITC excitation at $488 \mathrm{~nm}$ light, detected with a 500 to 530 bandpass filter; Cy5 excited at $633 \mathrm{~nm}$ and detected with a $650 \mathrm{~nm}$ longpass filter. Image acquisition was performed sequentially in the line modus to allow for detection of colocalization despite the rapid membrane movements. For line scan analysis, the membrane surfaces of 10 optical sections from each staining were selected and analyzed counterclockwise from the indicated starting points after using bandpass-filtered, color-adjusted, and merged images with the program WCIF-Image J Collection.

\section{Vectors, cloning, and sequencing}

NB1 was removed from clone pCMV-SPORT6-CD177 (RZPD, Berlin, Germany) by digestion with EcoRI and NotI (NEB, Frankfurt, Germany) and inserted into pcDNA4 (Invitrogen, Karlsruhe, Germany). pcDNA4- $\beta$ galactosidase (Invitrogen) was used for control transfection. All plasmids were sequenced using ABI PRISM 377 DNA sequencer (Applied Biosystems, Darmstadt, Germany) prior to use. Plasmids were amplified in Escherichia coli One Shot Top (Invitrogen) and purified by endotoxin-free Qiagen Maxi-Prep kit (Qiagen, Hilden, Germany).

\section{Cell culture and transfection}

HEK293 cells were cultivated in DMEM; HL60 cells were cultivated in RPMI supplemented with 10\% FCS, L-glutamine, and penicillin/ streptomycin (Biochrom, Berlin, Germany). HEK293 cells were transfected with FugeneHD transfection kit (Roche, Indianapolis, IN) and used 48 to 72 hours after transfection; HL60 cells were transfected using Amaxa Cell Line Nucleofactor Kit V (Amaxa Biosystems, Cologne, Germany) according to the manufacturer's instructions. Transfection efficiency was determined in each experiment and was greater than $80 \%$ for HEK293 cells and approximately $50 \%$ for HL60 cells. NB1- and control plasmid-transfected cells were incubated with $1 \mu \mathrm{g} / \mathrm{mL}$ (HEK293) or $5 \mu \mathrm{g} / \mathrm{mL}$ (HL60) purified neutrophil Proteinase 3 (The Binding Site, Birmingham, United Kingdom) and equimolar concentrations of myeloperoxidase (MPO) and human neutrophil elastase (HNE; Calbiochem, Nottingham, United Kingdom) for 2 hours on ice, washed, and stained for flow cytometry.

\section{Statistics}

Statistics were calculated using Stat view 4.5 (Abacus Concepts, Berkeley, CA). Correlations are given with $95 \%$ confidence interval. Values are given with SEM. For comparison of 2 groups, 2 -sided $t$ test was applied. $P$ values less than .05 were considered significant and are indicated by asterisks. 


\section{Results}

\section{Membrane PR3 expression highly correlates with membrane NB1 expression}

In previous studies, we had analyzed the neutrophil membrane expression of various molecules, including potential receptors, such as $\beta 2$-integrins and Fc $\gamma$ receptors. ${ }^{21}$ However, none of these molecules showed a bimodal surface expression pattern characteristic for PR3. The GPI-linked neutrophil glycoprotein NB1 has variable expression levels across the general population. ${ }^{17}$ Therefore, we tested for NB1 and PR3 membrane expression in parallel. Figure 1 shows expression in 2 healthy persons, one with $90 \%$ mPR3 expression (Figure 1A-B) and one negative for mPR3 (Figure 1C). The mNB1 percentages were identical, namely $90 \%$ and $0 \%$. The mean fluorescence for both proteins was increased after TNF- $\alpha$ priming in the $90 \%$ positive person (Figure 1A). We observed also a concentration-dependent mNB1 and mPR3 increase in response to fMLP (1B). fMLP $\left(10^{-9} \mathrm{M}\right)$ increased the MFI for NB1 to $170 \% \pm 35 \%$ and for PR3 to $141 \% \pm 15 \%$. fMLP at $10^{-8} \mathrm{M}$ resulted in further MFI increase to $230 \% \pm 32 \%$ for NB1 and to $223 \% \pm 52 \%$ for PR3. The values for fMLP at $10^{-6} \mathrm{M}$ were an MFI increase to $265 \% \pm 29 \%$ for NB1 and to $287 \% \pm 38 \%$ for PR3 $(n=3)$. However, stimulation did not affect the percentage of either mPR3 or mNB1-positive cells. Identical results were obtained with 4 different mAbs to PR3 (data not shown). By flow cytometry, we studied $\mathrm{mPR} 3$ and $\mathrm{mNB} 1$ expression in a total of 51 healthy donors and found a correlation of $r=0.996$ (Figure 1D). The same association was found in 11 patients with ANCAassociated vasculitis $(r=0.999$; Figure 1E).

\section{Membrane PR3-positive and membrane NB 1-positive neutrophils are identical}

Double staining was performed to analyze whether PR3 and NB1 were expressed on the surface of the same neutrophil subset. Cells staining positive for NB1 and PR3 were identical (Figure 2A). Stainings for the GPI-linked surface molecules CD66b and CD16b served as controls and showed no difference in fluorescence intensity between the mPR3-positive and -negative subsets (Figure 2A). As a second approach, we separated mPR3-positive and -negative neutrophils by magnetic cell sorting and determined mNB1 expression in these physically separated cell populations. NB1 was only detected on the cells positively sorted for mPR3 (Figure 2B). In contrast, no NB1 membrane staining was observed on neutrophils without mPR3 expression. Together, both experimental approaches independently demonstrate identity of mPR3 and mNB1-expressing neutrophils.

\section{Membrane PR3 expression is restricted to cells with NB1}

We next assayed intracellular PR3 and NB1 by Western blot in neutrophils sorted for membrane expression of either PR3 or NB1. We detected PR3 in all neutrophil lysates, independent of cell membrane expression (Figure 3). In contrast, NB1 was only found in lysates of neutrophils selected for positive membrane staining for PR3 or NB1. Western blot also demonstrated that there was no cross-reactivity of the used antibodies. These data support the hypothesis that the presence of NB1 is the determinate of membrane PR3 expression by acting as a receptor for display.
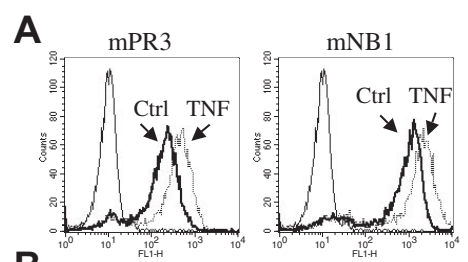

B

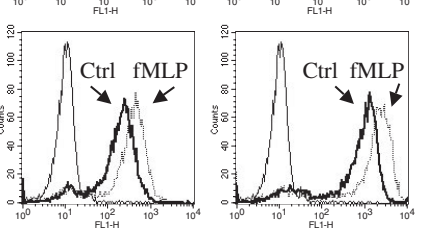

C
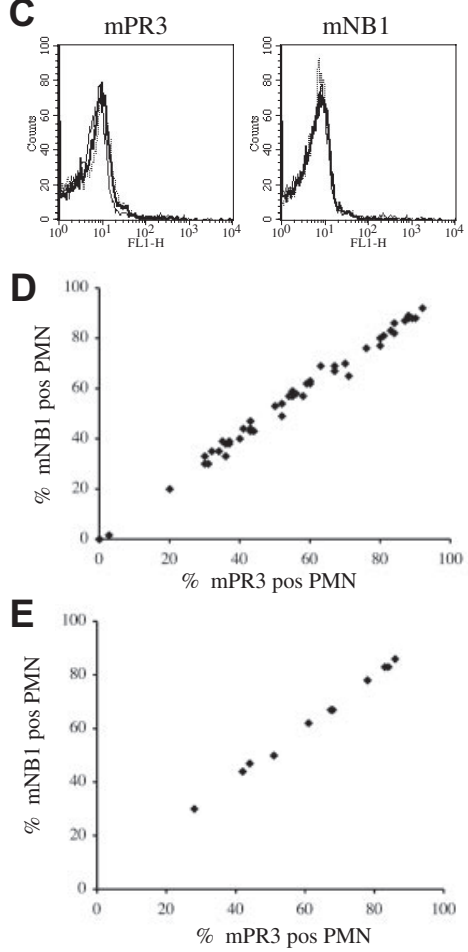

Figure 1. Membrane PR3 expression highly correlates with membrane expression of granulocyte antigen NB1. Live neutrophils were surface stained with monoclonal antibodies. Fluorescence was measured by flow cytometry. Percentages of membrane PR3- and NB1-positive cells were identical in donors with different percentages of membrane-positive cells (A-B, $90 \% ; \mathrm{C}, 0 \%$ ). MFI of both proteins increased after stimulation with TNF- $\alpha$ (A; bold line for cells stimulated with buffer control and dotted line for TNF- $\alpha$ stimulation) with $\mathrm{MLLP}$ (bold line for buffer control, dashed line for $10^{-6} \mathrm{M}$ fMLP). In contrast, the NB1- and PR3-positive percentage remained unchanged, and no increase in the expression of either molecule occurred with TNF- $\alpha$ when cells from an mPR3/NB1-negative donor were used (C). (D) The correlation is shown of PR3 and NB1 percentages in 51 healthy donors $(r=0.996$, $P<.001$ ). (E) The values are given for patients with ANCA-associated vasculitis $(r=0.999, P<.001)$.

\section{Membrane NB1 and membrane PR3 expression correlate in stem-cell-derived neutrophils}

To study mPR3 and mNB1 expression during neutrophil development, we used a model of in vitro differentiation from umbilical CD $34^{+}$hematopoietic stem cells as described earlier. ${ }^{11}$ Differentiation was assessed by morphology and neutrophil surface marker expression (Figure 4). Identical mPR3- and mNB1-positive percentages were detected after 7 days of differentiation. These experiments indicate that differentiation of neutrophils, even under in vitro conditions, results in coexpression of PR3 and NB1 on a proportion of cells. Furthermore, they strengthen the contention that the GPI-linked NB1 receptor molecule is causally involved in mPR3 presentation. 


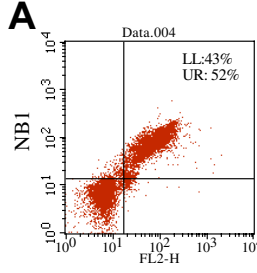

PR3

B

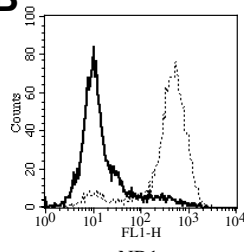

NB1

Figure 2. Membrane PR3-positive and NB1-positive neutrophils are identical. Neutrophils were stained with anti-PR3 and PE-labeled secondary antibody ( $x$-axis) and FITC-labeled antibodies against NB1, CD16b, and CD66b (A). Measurements were taken after the appropriate compensation. Percentages of cells in the quadrants are noted. (B) Neutrophil membrane PR3-positive and -negative subpopulations were separated by magnetic cell sorting and stained with directly labeled anti-NB1 antibody. mPR3-negative cells do not stain for NB1 (bold line), whereas mPR3positively sorted cells are recognized by the anti-NB1 antibody (dotted line).

\section{Loss of GPI linkers from plasma membrane of neutrophils results in decreased $\mathrm{mPR} 3$ expression}

NB1 is a GPI-linked protein and can be removed from the cell membrane by cleaving the glucosamine moiety of the GPI linker

A

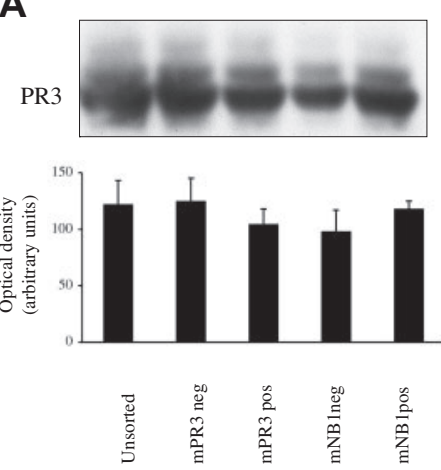

B NB1
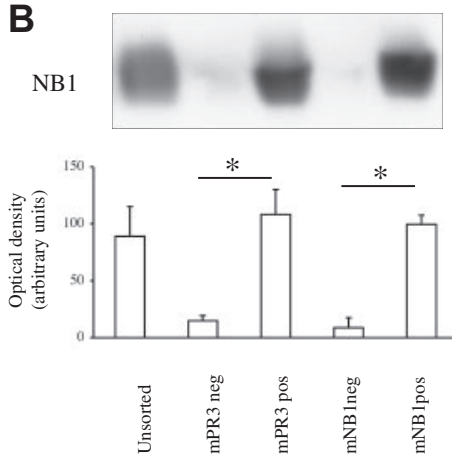

Figure 3. Membrane PR3 expression is restricted to cells with NB1 protein. Cells sorted for mPR3 $(n=5, A)$ and mNB1 $(n=3$, B) expression (purity, $90 \% \pm 5 \%$ ) were subjected to immunoblot analysis for PR3 and NB1. Corresponding optical densitometry showed no significant difference in whole-cell PR3 content in the membrane-positive and -negative subpopulations. However, NB1 was restricted to mPR3- and mNB1-positive cells $\left({ }^{*} P<.05\right)$. Error bars indicate \pm SEM.

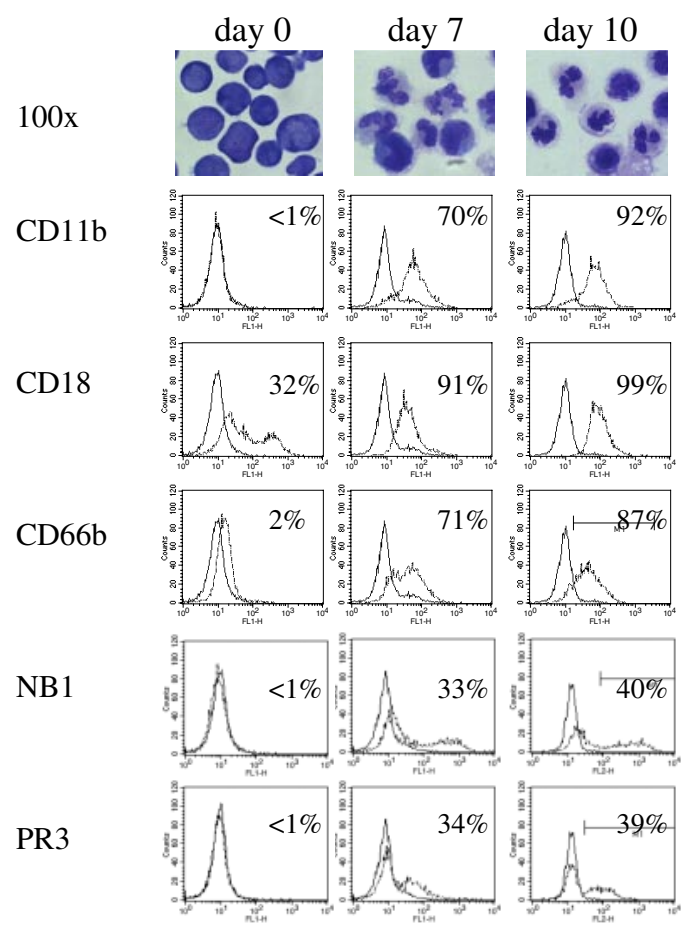

Figure 4. NB1 and PR3 surface expression in an in vitro model of neutrophil differentiation. $\mathrm{CD}_{3}{ }^{+}$stem cells were isolated from umbilical cord blood, expanded, and differentiated into neutrophils. Morphology is shown using WrightGiemsa staining and microscopy. Surface expression of differentiation markers was assessed by flow cytometry. Seven days of differentiation resulted in identical PR3 and NB1 percentages as shown for a typical of 10 independent experiments. from its lipid membrane anchor using phospholipase C. ${ }^{22}$ Neutrophils treated with phospholipase $C$ showed a reduction of mNB1 expression in flow cytometry (Figure 5A-B). This reduction was accompanied by decreased mPR3 expression, whereas the control marker CD18 was unaffected. In addition, we studied neutrophils from a patient with paroxysmal nocturnal hemoglobinuria $(\mathrm{PNH})$, a rare acquired defect in the formation of GPI linkers. ${ }^{23}$ These patients show a variable decrease of GPI-linked proteins on their neutrophils. ${ }^{24}$ In parallel to a healthy donor we document that the neutrophils of the patient with PNH had a major decrease in a number of GPI-linked proteins (Figure 5C). No NB1 was detected on their surface, although intracellular NB1 was present in Western blot (data not shown). Similar to the NB1 results, PR3 was also absent from the cell surface. These experiments further suggest NB1 as a receptor that is necessary for PR3 membrane presentation.

\section{PR3 and NB1 colocalize on and are coimmunoprecipitated from the neutrophil plasma membrane}

Live-cell confocal microscopy shows that PR3 and NB1 were not only localized on the same cell but also colocalized on the plasma membrane (Figure 6A; Figure S1, available on the Blood website; see the Supplemental Materials link at the top of the online article). We did not observe similar proximity between mPR3 and CD66b or CD16b. We were also able to coimmunoprecipitate NB1 and PR3 from the supernatant of neutrophils that were treated with phospholipase $\mathrm{C}$ to mobilize GPI-linked proteins from the plasma membrane (Figure 6B). These results demonstrate physical interac- 
Figure 5. PR3 neutrophil membrane display depends on GPI linkers. Neutrophils subjected to GPI-anchor cleavage by phospholipase $C$ showed decreased membrane NB1 and PR3 expression (A, dotted lines) as compared with control cells (bold lines). The mean change of fluorescence in 7 independent experiments was $-43 \% \pm 4 \%$ for NB1 and $-33 \% \pm 12 \%$ for PR3, $P<.05$. Error bars indicate SEM; ${ }^{\star} P<.05$; ${ }^{* \star} P<.01$. (B). The $\beta 2$-integrin CD18, a marker of cell activation, remained unchanged $(6 \pm 6)$. (C) NB1 and several other GPI-linked proteins are expressed on neutrophils from a patient with $\mathrm{PNH}$ in comparison to a healthy control subject. Isotype control antibody staining is shown by the thin line and the GPI-linked molecule staining by bold lines. $\mathrm{PNH}$ is characterized by an acquired deficiency in GPI-linker synthesis. Membrane display of GPI-linked proteins was strongly decreased, with $\mathrm{mNB} 1$ and $\mathrm{mPR} 3$ expression even being absent in the patient was $\mathrm{PNH}$.
$A_{\text {of }}$
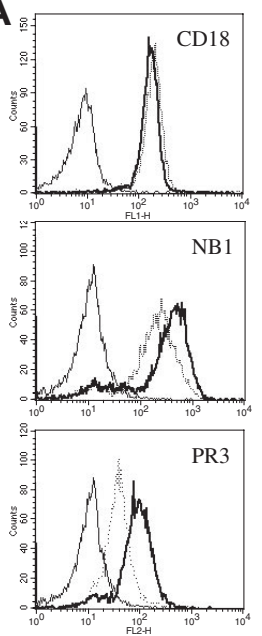

B

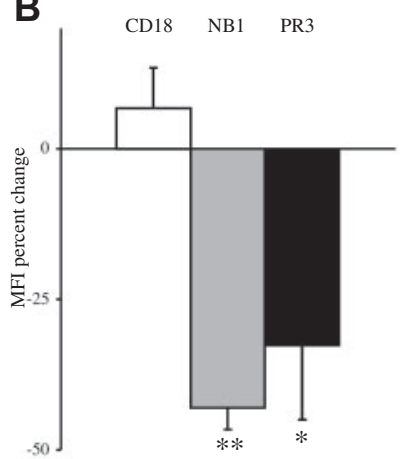

C

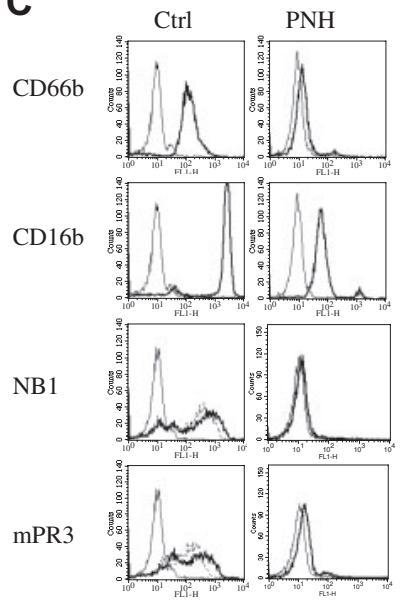

tion of PR3 and NB1 on the neutrophil plasma membrane as expected for a receptor-ligand pair.

\section{NB1 transfection results in NB1 membrane expression and enables PR3 binding}

NB1 transfected into HEK293 cells resulted in correct-sized protein expression in Western blot analysis (data not shown), and surface NB1 expression was demonstrated by flow cytometry (Figure 7A). NB1-transfected cells that were incubated with purified human PR3 showed markedly elevated PR3 surface display compared with mock-transfected cells. HEK293 cells were incubated with control proteins at equimolar concentrations in parallel. We selected the second major ANCA antigen myeloperoxidase (MPO) and human neutrophil elastase (HNE), another neutrophil protease of similar size as PR3. Our data show that neither MPO nor HNE surface display was increased by NB1 expression (Figure 7B). Similar results were obtained after NB1 transfection into HL60 cells, a myeloblastic cell line (Figure S2). These data demonstrate that NB1 confers the ability to display PR3 on the plasma membrane.

\section{Discussion}

Our study is the first to show that NB1, a GPI-linked receptor, is necessary for the presentation of the ANCA autoantigen PR3 on the membrane surface of human neutrophils. Autoantibody binding to mPR3 is characteristic for ANCA-associated vasculitis ${ }^{25}$ and results in cell activation with release of reactive oxygen species and tissue-toxic granule proteins. ${ }^{2,26}$ Experimental data suggest that mPR3-positive neutrophils show a stronger respiratory burst activity when incubated with PR3-ANCA than mPR3-negative cells from the same donor. ${ }^{21}$ Understanding how PR3 is displayed on the neutrophil could have mechanistic and therapeutic importance.

The mechanism of PR3 presentation on the cell surface has not yet been defined. On endothelial cells, a 111-kDa protein was suggested to serve as a PR3 receptor, but the nature of this molecule has not been shown. ${ }^{27}$ In neutrophils, an association of mPR3 with the $\beta 2$-integrins CD18 and CD11b and with the Fc $\gamma$-receptor IIIB in lipid rafts was described..$^{14,28}$ However, none of these molecules show a bimodal membrane phenotype that is characteristic for

A
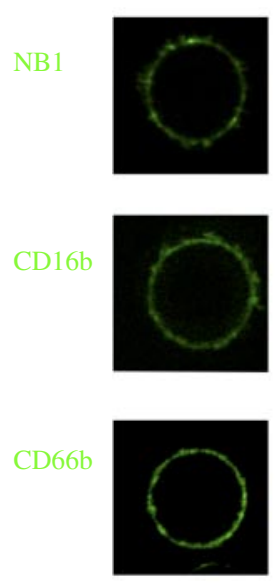

PR3
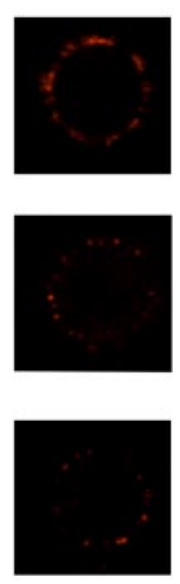

Merge
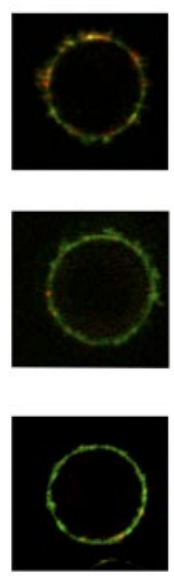

line scan
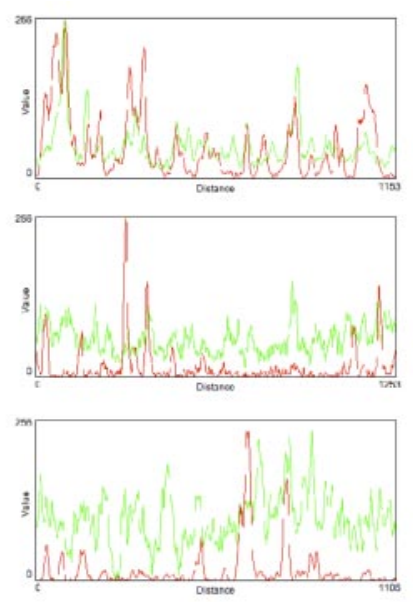

B
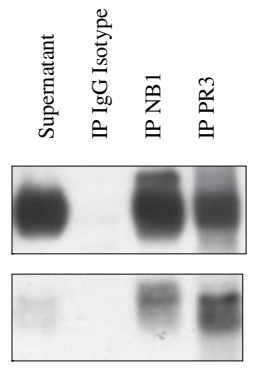

NB1

PR3

Figure 6. PR3 and NB1 colocalize on and coimmunoprecipitate from the neutrophil plasma membrane. Confocal microscopy of live neutrophils showed colocalization of PR3- and NB1-stained spots on the plasma membrane in comparison to CD66b and CD16b (A). Line scans of fluorescence intensity indicate colocalization of peak color intensities. Immunoprecipitation and Western blot analysis of PR3 and NB1 was performed from the supernatant of TNF- $\alpha$-primed, phospholipase C-treated neutrophils. This procedure resulted in specific coimmunoprecipitation of PR3 after NB1 precipitation and vice versa (B). 
A

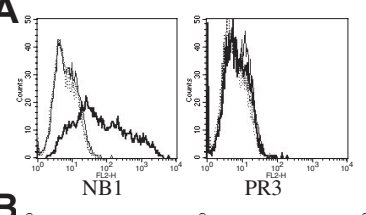

B
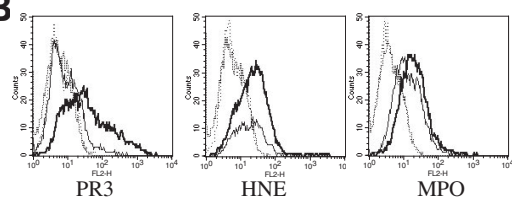

C

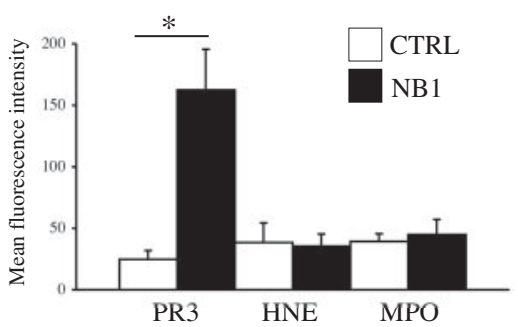

Figure 7. Ectopic NB1 expression confers PR3 surface binding. Transfection of a NB1 expression plasmid into HEK293 cells resulted in NB1 surface expression (A; bold line for NB1-transfected cells, thin line for ctrl-transfected cells, isotype control staining for ctrl- and NB1-transfection is depicted as dotted lines). Monoclonal anti-PR3 antibodies did not bind to any of these cells as shown for CLB12.8. (B) NB1 transfected (bold line) and mock-transfected cells (thin line) were incubated on ice for 2 hours with $1 \mu \mathrm{g} / \mathrm{mL}$ purified PR3 or equimolar concentrations of neutrophil elastase (HNE) or myeloperoxidase (MPO), washed and stained for surface display. (C) Binding of PR3 was specifically elevated in NB1-transfected cells $(\square)$ as compared with mock-transfected $(\square)$ cells (MFI, $163 \pm 33$ versus $25 \pm 7 ;{ }^{\star} P<.05 ; n=5$ independent transfections). HNE and MPO binding was unaffected by NB1 transfection (MFI: HNE, $36 \pm 10$ versus $39 \pm 16$, and MPO, $45 \pm 12$ versus $39 \pm 6 ; n=3$ independent transfections). Error bars indicate SEM.

mPR3. Our studies demonstrate that mNB1 expression has characteristics similar to PR3. Both molecules show bimodal expression patterns, and cytokine-priming increases the amount of surface expression without changing the percentage of positive cells. Similar findings were recently described in a prepublished manuscript by Bauer et al. ${ }^{29}$ In addition, we show by several independent methods in isolated blood neutrophils and in vitro-differentiated stem cell-derived neutrophils that the neutrophil subset that expresses surface NB1 and PR3 is the same.

$\mathrm{NB} 1$ is a neutrophil surface receptor that was described originally as a neutrophil alloantigen. Similar to $\mathrm{mPR} 3$, the percentage of $\mathrm{mNB} 1-$ positive neutrophils ranges from $0 \%$ to $100 \%$. The percentage was stable in healthy persons and did not change with G-CSF administration. ${ }^{30,31} \mathrm{NB} 1$ polymorphisms were described, and defective splicing was proposed as a mechanism explaining the phenotype in $0 \%$ NB1-expressing persons. ${ }^{32,33}$ Acquired anti-NB1 antibodies in a NB1deficient mother during a previous pregnancy were responsible for neonatal neutropenia after consecutive pregnancies. ${ }^{15} \mathrm{~A}$ second disease association was found with PRV, where the NB1 mRNA expression was increased in peripheral blood neutrophils. ${ }^{34}$ The protein was therefore also named PRV1 and only later found to be an allelic variant of NB1. ${ }^{35}$ NB1 belongs to the same family of GPI-linked molecules as the receptor of another serine-protease, namely urokinase-type plasminogen activator receptor (uPAR).

Live-cell confocal microscopy and coprecipitation from the membrane was used to demonstrate that both molecules indeed physically interacted. Other GPI-linked receptors, such as CD16b and CD66b, did not demonstrate the same degree of colocalization. Enzymatic removal of GPI linkers off the neutrophil membrane resulted in decreased mPR3 expression. In addition, we had the opportunity to study neutrophils from a patient with $\mathrm{PNH}$, an acquired clonal loss of GPI linker synthesis. ${ }^{24}$ Our patient showed the expected reduction of CD66b and $\mathrm{CD} 16 \mathrm{~b}$, and even loss of NB1 with a parallel lack of mPR3 expression on neutrophils. To provide firm evidence that NB1 expression confers the capability to express mPR3, we transfected different cell lines with NB1. Transgenic NB1 membrane expression resulted in the ability to bind exogenous human PR3, whereas the control proteins HNE and MPO did not show specific binding.

Our data establish the NB1 receptor as the mechanism of mPR3 display. Our findings also raise a number of new and interesting questions. We show that exogenous PR3 binds to mNB1 and also find a parallel membrane up-regulation of both molecules in response to proinflammatory degranulation-inducing mediators. However, whether NB1 and PR3 are also translocated as a complex from intracellular pools, such as secondary granules or secretory vesicles is currently unknown. Both granule types were shown to contain NB1 and PR3. Furthermore, it is also conceivable that PR3 and NB1 translocate independently and associate on the membrane. Failed efforts of coimmunoprecipitation from secondary vesicles (S.v.V., unpublished data, April-July, 2006) favor the latter explanation, but more detailed investigations are needed to clarify intracellular association.

No physiologic ligand of NB1 has been described, and little is known on signal transduction by this molecule. However, anti-NB1 antibodies were shown to induce respiratory burst activity in primed neutrophils, similar to PR3-ANCA. ${ }^{36}$ In fact, we obtained data indicating that anti-NB1 mAb result in phosphorylation of $\mathrm{p} 38$ MAPK, ERK, and PI3K/Akt (data not shown). Other investigators and our own group showed previously that all 3 of these pathways control ANCA-induced neutrophil activation. ${ }^{37-39}$ Possibly, additional molecules are recruited to the NB1-PR3 complex before neutrophils are activated. The UPAR is a well-studied member of the same family that plays an important role in coagulation and cell migration. ${ }^{40}$ For signaling, uPAR associates with integrins as was shown in epithelial and mesenchymal cells. ${ }^{41,42} \beta 2$-Integrins are important in neutrophil signaling; their association with mPR3 was reported earlier. ${ }^{14,26,43}$ It is tempting to speculate that a complex of PR3 and NB1 with integrins and possibly Fc $\gamma$-receptor forms on PR3-ANCA binding and mediates PR3-ANCA-induced neutrophil activation. ${ }^{28,44}$ This issue will be the subject of further investigations that will require a neutrophil model system that can be successfully transfected and subjected to activation studies.

In summary, we show that PR3 is presented on human neutrophils by the surface receptor NB1. This finding identifies $\mathrm{NB} 1$ as a potential therapeutic target in ANCA-associated vasculitis.

\section{Acknowledgments}

This work was supported by InnoRegio 03 I 4509B, ProFIT (R.K.), and the European Union ESF program (G.T.).

\section{Authorship}

Contribution: S.v.V. designed and performed research and wrote the paper; G.T. and C.E. performed research; M.W. and M.C.C. supervised and designed research; and F.C.L. and R.K. designed research and wrote the paper. All authors approved the manuscript.

Conflict-of-interest disclosure: The authors declare no competing financial interests.

Correspondence: Ralph Kettritz, Nephrology and Hypertension Section, Franz-Volhard-Klinik, Wiltbergstrasse 50, 13125 Berlin, Germany; e-mail: kettritz@charite.de. 


\section{References}

1. Campanelli D, Melchior M, Fu Y, et al. Cloning of cDNA for proteinase 3: a serine protease, antibiotic, and autoantigen from human neutrophils. $J$ Exp Med. 1990;172:1709-1715.

2. Falk RJ, Terrell RS, Charles LA, Jennette JC. Anti-neutrophil cytoplasmic autoantibodies induce neutrophils to degranulate and produce oxygen radicals in vitro. Proc Natl Acad Sci U S A. 1990;87:4115-4119.

3. Pfister H, Ollert M, Frohlich LF, et al. Antineutrophil cytoplasmic autoantibodies against the murine homolog of proteinase 3 (Wegener autoantigen) are pathogenic in vivo. Blood. 2004;104: 1411-1418.

4. Bories D, Raynal MC, Solomon DH, Darzynkiewicz Z, Cayre YE. Down-regulation of a serine protease, myeloblastin, causes growth arrest and differentiation of promyelocytic leukemia cells. Cell. 1989;59:959-968.

5. Goldschmeding R, van der Schoot CE, ten Bokkel Huinink D, et al. Wegener's granulomatosis autoantibodies identify a novel diisopropylfluorophosphate-binding protein in the lysosomes of normal human neutrophils. J Clin Invest. 1989;84: 1577-1587.

6. Halbwachs-Mecarelli L, Bessou G, Lesavre P, Lopez S, Witko-Sarsat V. Bimodal distribution of proteinase 3 (PR3) surface expression reflects a constitutive heterogeneity in the polymorphonuclear neutrophil pool. FEBS Lett. 1995;374:2933.

7. Schreiber A, Busjahn A, Luft FC, Kettritz R. Membrane expression of proteinase 3 is genetically determined. J Am Soc Nephrol. 2003;14:68-75.

8. von Vietinghoff S, Busjahn A, Schonemann C, et al. Major histocompatibility complex HLA region largely explains the genetic variance exercised on neutrophil membrane proteinase 3 expression. J Am Soc Nephrol. 2006;17:3185-3191.

9. Abdgawad M, Hellmark T, Gunnarsson L, Westman KW, Segelmark M. Increased neutrophil membrane expression and plasma level of proteinase 3 in systemic vasculitis are not a consequence of the $-564 \mathrm{~A} / \mathrm{G}$ promotor polymorphism Clin Exp Immunol. 2006;145:63-70.

10. Rarok AA, Stegeman CA, Limburg PC, Kallenberg CG. Neutrophil membrane expression of proteinase 3 (PR3) is related to relapse in PR3ANCA-associated vasculitis. J Am Soc Nephrol. 2002; 13:2232-2238

11. Schreiber A, Otto B, Ju X, et al. Membrane proteinase 3 expression in patients with Wegener's granulomatosis and in human hematopoietic stem cell-derived neutrophils. J Am Soc Nephrol. 2005;16:2216-2224

12. Witko-Sarsat $V$, Lesavre $P$, Lopez $S$, et al. A large subset of neutrophils expressing membrane proteinase 3 is a risk factor for vasculitis and rheumatoid arthritis. J Am Soc Nephrol. 1999;10: 1224-1233.

13. Goldmann WH, Niles JL, Arnaout MA. Interaction of purified human proteinase 3 (PR3) with reconstituted lipid bilayers. Eur J Biochem. 1999;261: 155-162.

14. David A, Kacher $Y$, Specks $U$, Aviram I. Interaction of proteinase 3 with CD11b/CD18 (beta2 integrin) on the cell membrane of human neutrophils. J Leukoc Biol. 2003;74:551-557.

15. Lalezari P, Murphy GB, Allen FH Jr. NB1, a new neutrophil-specific antigen involved in the pathogenesis of neonatal neutropenia. J Clin Invest. 1971;50:1108-1115.

16. Klippel S, Strunck E, Busse CE, Behringer D, Pahl HL. Biochemical characterization of PRV-1, a novel hematopoietic cell surface receptor, which is overexpressed in polycythemia rubra vera. Blood. 2002;100:2441-2448.

17. Stroncek DF, Caruccio L, Bettinotti M. CD177: a member of the Ly- 6 gene superfamily involved with neutrophil proliferation and polycythemia vera. J TransI Med. 2004;2:8.

18. Stroncek D. Neutrophil-specific antigen HNA-2a (NB1, CD177): serology, biochemistry, and molecular biology. Vox Sang. 2002;83(suppl 1):359361.

19. Jennette JC, Falk RJ, Andrassy K, et al. Nomenclature of systemic vasculitides. Proposal of an international consensus conference. Arthritis Rheum. 1994;37:187-192.

20. Leavitt RY, Fauci AS, Bloch DA, et al. The American College of Rheumatology 1990 criteria for th classification of Wegener's granulomatosis. Arthritis Rheum. 1990;33:1101-1107.

21. Schreiber A, Luft FC, Kettritz R. Membrane proteinase 3 expression and ANCA-induced neutrophil activation. Kidney Int. 2004;65:2172-2183.

22. Skubitz KM, Stroncek DF, Sun B. Neutrophil-specific antigen NB1 is anchored via a glycosyl-phosphatidylinositol linkage. J Leukoc Biol. 1991;49: 163-171.

23. Rosti V. The molecular basis of paroxysmal nocturnal hemoglobinuria. Haematologica. 2000;85: 82-87.

24. Edberg JC, Salmon JE, Whitlow M, Kimberly RP. Preferential expression of human Fc gamma RIIIPMN (CD16) in paroxysmal nocturnal hemoglobinuria. Discordant expression of glycosyl phosphatidylinositol-linked proteins. J Clin Invest. 1991;87:58-67.

25. van der Woude FJ, Rasmussen N, Lobatto S, et al. Autoantibodies against neutrophils and monocytes: tool for diagnosis and marker of disease activity in Wegene's granulomatosis. Lancet. 1985;1:425-429.

26. Reumaux D, Vossebeld PJ, Roos D, Verhoeven AJ. Effect of tumor necrosis factor-induced integrin activation on Fc gamma receptor II-mediated signal transduction: relevance for activation of neutrophils by anti-proteinase 3 or anti-myeloperoxidase antibodies. Blood. 1995;86:3189-3195.

27. Taekema-Roelvink ME, Van Kooten C, Heemskerk E, Schroeijers W, Daha MR. Proteinase 3 interacts with a 111-kD membrane molecule of human umbilical vein endothelial cells. J Am Soc Nephrol. 2000;11:640-648.

28. Fridlich R, David A, Aviram I. Membrane proteinase 3 and its interactions within microdomains of neutrophil membranes. J Cell Biochem. 2006;99: 117-125

29. Bauer S, Abdgawad M, Gunnarsson L, Segelmark M, Tapper H, Hellmark T. Proteinase 3 and CD177 are expressed on the plasma membrane of the same subset of neutrophils. J Leukoc Biol. 2007;81:458-464.

30. Goldschmeding R, van Dalen CM, Faber N, et al Further characterization of the NB 1 antigen as a variably expressed 56-62 kD GPI-linked glycoprotein of plasma membranes and specific gran- ules of neutrophils. Br J Haematol. 1992;81:336345.

31. Wolff J, Brendel C, Fink L, Bohle RM, Kissel K Bux J. Lack of NB1 GP (CD177/HNA-2a) gene transcription in NB1 GP- neutrophils from NB1 GP-expressing individuals and association of low expression with NB1 gene polymorphisms. Blood. 2003;102:731-733.

32. Caruccio L, Walkovich K, Bettinotti M, Schuller R, Stroncek D. CD177 polymorphisms: correlation between high-frequency single nucleotide polymorphisms and neutrophil surface protein expression. Transfusion. 2004;44:77-82.

33. Kissel K, Scheffler S, Kerowgan M, Bux J. Molecular basis of NB1 (HNA-2a, CD177) deficiency. Blood. 2002;99:4231-4233.

34. Temerinac S, Klippel S, Strunck E, et al. Cloning of PRV-1, a novel member of the uPAR receptor superfamily, which is overexpressed in polycythemia rubra vera. Blood. 2000;95:2569-2576.

35. Bettinotti MP, Olsen A, Stroncek D. The use of bioinformatics to identify the genomic structure of the gene that encodes neutrophil antigen NB1, CD177. Clin Immunol. 2002;102:138-144.

36. Bartunkova J, Araujo A, Hrusak O, Sediva A. Autoimmunity to polymorphonuclears: functional consequences of the binding of antibodies to membrane and cytoplasmic target antigens of polymorphonuclear leukocytes. J Clin Immunol. 1997; 17:455-461.

37. Ben-Smith A, Dove SK, Martin A, Wakelam MJ, Savage CO. Antineutrophil cytoplasm autoantibodies from patients with systemic vasculitis activate neutrophils through distinct signaling cascades: comparison with conventional Fcgamma receptor ligation. Blood. 2001;98:1448-1455.

38. Kettritz R, Scheumann J, Xu Y, Luft FC, Haller H TNF-alpha-accelerated apoptosis abrogates ANCA-mediated neutrophil respiratory burst by a caspase-dependent mechanism. Kidney Int. 2002;61:502-515

39. Kettritz R, Schreiber A, Luft FC, Haller H. Role of mitogen-activated protein kinases in activation of human neutrophils by antineutrophil cytoplasmic antibodies. J Am Soc Nephrol. 2001;12:37-46.

40. Ragno P. The urokinase receptor: a ligand or a receptor? Story of a sociable molecule. Cell Mol Life Sci. 2006;63:1028-1037

41. Franco P, Vocca I, Carriero MV, et al. Activation of urokinase receptor by a novel interaction between the connecting peptide region of urokinase and alpha v beta 5 integrin. J Cell Sci. 2006;119: 3424-3434.

42. Ghosh S, Johnson JJ, Sen R, et al. Functional relevance of urinary-type plasminogen activator receptor-alpha3beta1 integrin association in proteinase regulatory pathways. J Biol Chem. 2006; 281:13021-13029.

43. Kettritz R, Choi M, Rolle S, Wellner M, Luft FC Integrins and cytokines activate nuclear transcription factor-kappaB in human neutrophils. J Biol Chem. 2004;279:2657-2665.

44. Williams JM, Ben-Smith A, Hewins P, et al. Activation of the $G(i)$ heterotrimeric $G$ protein by ANCA IgG $F\left(a b^{\prime}\right) 2$ fragments is necessary but not sufficient to stimulate the recruitment of those downstream mediators used by intact ANCA IgG. J Am Soc Nephrol. 2003;14:661-669. 

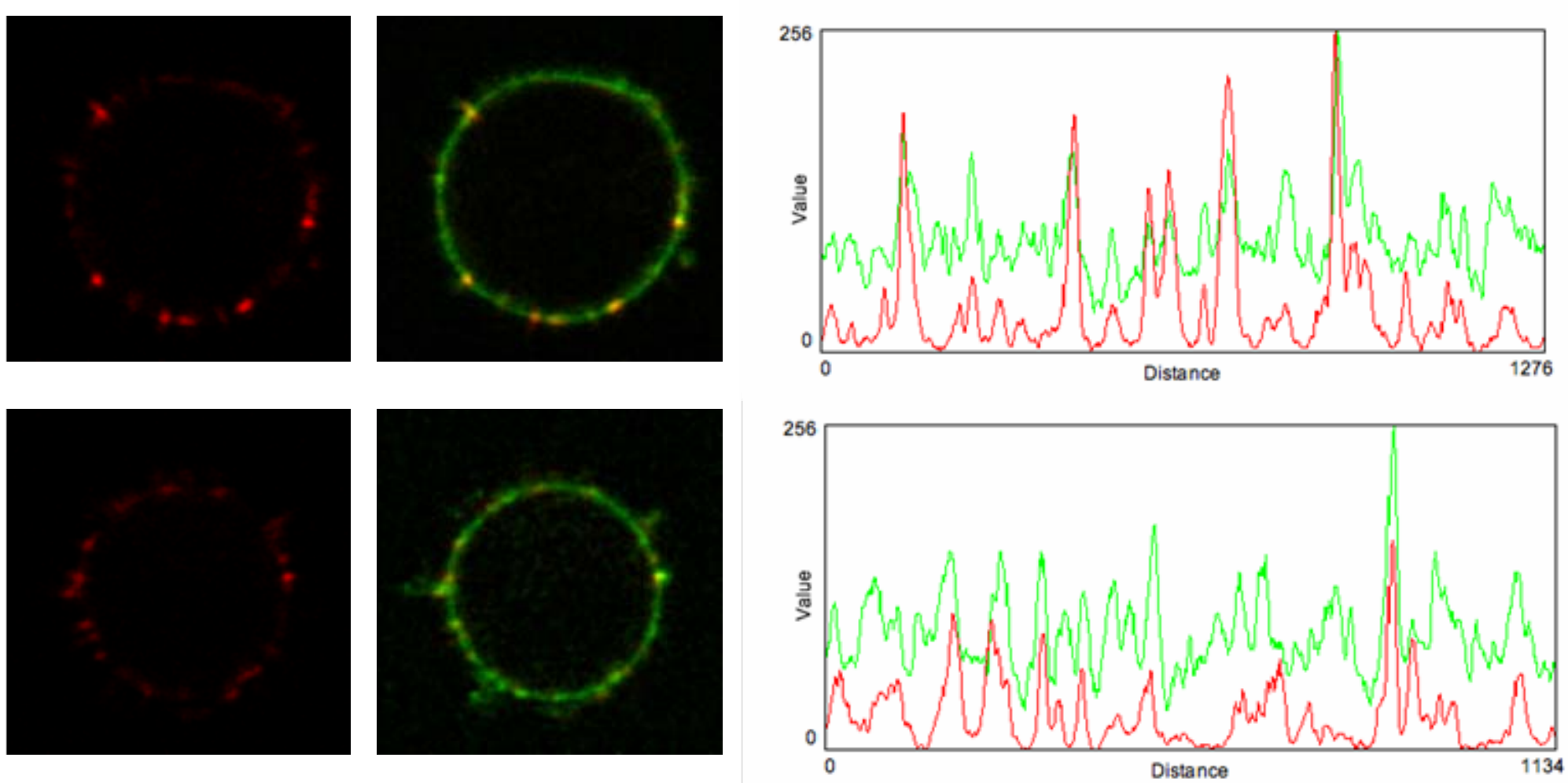


\section{Ctrl-transfected}
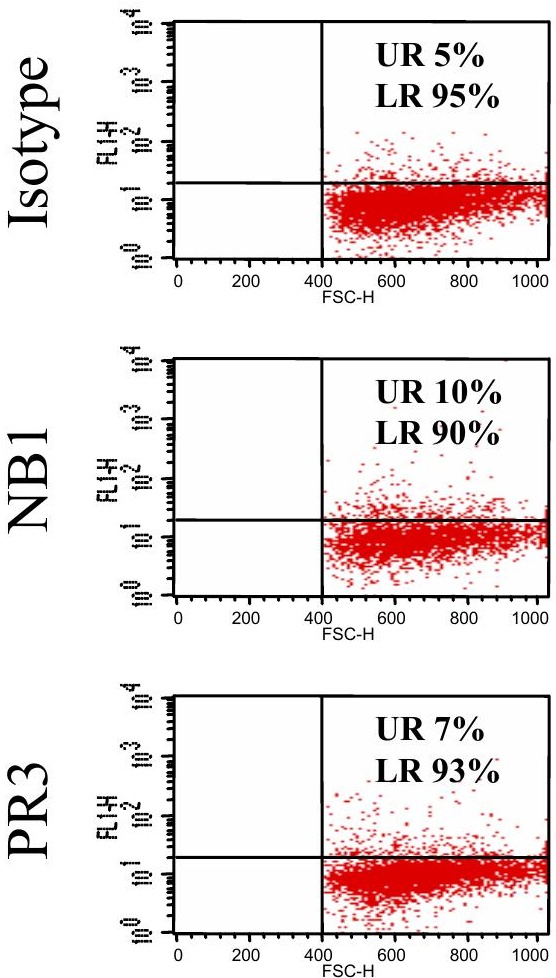

NB1-transfected
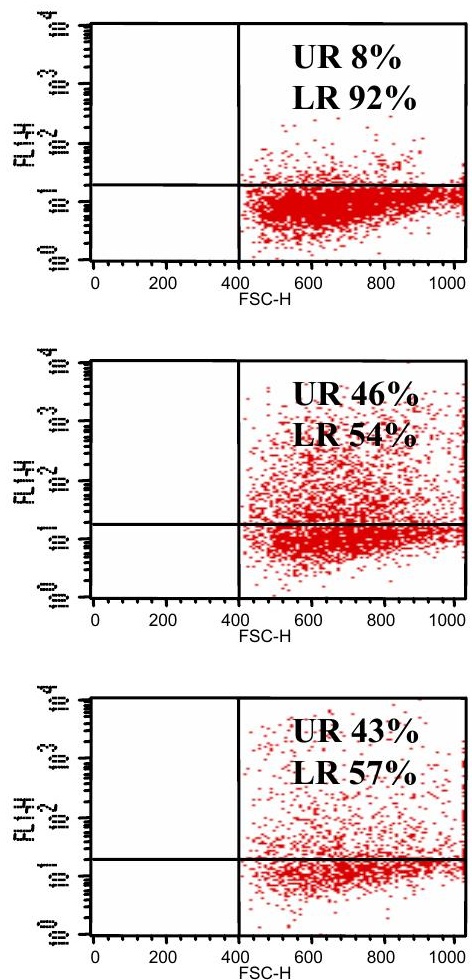